Résumés des conférences et travaux

\title{
Histoire et philologie de la Chine classique
}

\section{François Martin}

\section{OpenEdition \\ Journals}

\section{Édition électronique}

URL : https://journals.openedition.org/ashp/1217

DOI : $10.4000 /$ ashp. 1217

ISSN : 1969-6310

Éditeur

Publications de l'École Pratique des Hautes Études

\section{Édition imprimée}

Date de publication : 1 octobre 2011

Pagination : 256-265

ISSN : 0766-0677

\section{Référence électronique}

François Martin, "Histoire et philologie de la Chine classique ". Annuaire de l'École pratique des hautes études (EPHE), Section des sciences historiques et philologiques [En ligne], 142 | 2011, mis en ligne le 23 septembre 2011, consulté le 06 juillet 2021. URL : http://journals.openedition.org/ashp/1217 ; DOI : https://doi.org/10.4000/ashp.1217 


\title{
HISTOIRE ET PHILOLOGIE DE LA CHINE CLASSIQUE
}

\author{
Directeur d'études : M. François MARTIN
}

Programme de l'année 2008-2009 : I. Gens du Nord et gens du Sud : textes des Six Dynasties. - II. Une capitale de la naissance à la mort : Nankin sous les Six Dynasties (suite).

\section{Gens du Nord et gens du Sud : textes des Six Dynasties}

Après nous être consacré l'année précédente à quelques cas de transfuges notoires, on a commencé cette année l'étude de deux cas d'empereurs « fous », l'un au Nord, l'autre au Sud. On a pu seulement étudier le « cas septentrional», remettant le « cas méridional » à l'année suivante. On s'est souvent interrogé, à propos de nombreux empereurs romains qui se sont distingué par leur dépravation et leur cruauté : étaientils des malades mentaux ou bien est-ce le pouvoir dont ils jouissaient qui les avait pervertis? L'histoire de Chine, et singulièrement sous les Six Dynasties, nous fournit un assez grand nombre de cas sur lesquels on peut se poser la même question. Sans avoir la prétention d'y apporter une réponse générale, sans doute impossible, on s'est penché cette année sur la personne de Gao Yang 高洋 (529, regnavit 550-559), premier empereur des Qi du Nord 北齊, dont la vie nous est bien connue par le Livre des Qi septentrionaux (Bei-Qi shu 北齊書) de Li Baiyao 李百藥 (565-648), historien de peu postérieur à Gao Yang et qui fut à même de recueillir de ses aînés des informations encore assez récentes. C'est en 629 que Li Baiyao reçut de l'empereur Taizong des Tang l'ordre de composer cet ouvrage, qu'il acheva en 636. Cependant, ce n'est pas de son livre que nous sommes partis, mais du Miroir général pour aider au gouvernement (Zizhi tongjian 資質通鑑) du célèbre historien Song Sima Guang 司馬光 (10191086). Comme on le sait, ce dernier a arrangé ses sources selon un ordre chronologique commode et particulièrement adapté à une étude telle que celle que nous nous proposions de faire. On a cependant toujours eu soin de confronter son texte à celui de l'Histoire des Qi septentrionaux, qu'il a parfois légèrement abrégé ou modifié.

En traitant Gao Yang de fou sanguinaire, nous n'avons rien découvert. Withold Rodzinski a dit des souverains des Qi du Nord qu'ils avaient été peut-être « les monstres les plus fantastiquement dégénérés dans la longue ligne des mauvais souverains de la Chine ${ }^{1}$ ». De Gao Yang en particulier, Arthur Wright a dit : " Gao Yang se révéla comme l'un des plus effarants tyrans de l'histoire de Chine. Sa soif de sang, sa cruauté insatiable et sa mégalomanie firent de son règne une horreur ininterrompue ${ }^{2}$. » Récemment enfin, Jennifer Holmgren lui a consacré un article intitulé « Seeds of Madness ${ }^{3}$ ». Pourtant, Gao Yang témoigna tôt d'une vive intelligence. Il en fit preuve à plusieurs reprises, en particulier un jour que son père, le fameux chef de guerre Gao Huan

1. A History of China, New York, 1079, t. I, p. 99.

2. The Sui Dynasty, New York, 1978, p. 38.

3. Papers on Far-Eastern History 24, 1081, p. 81-134. 
高歡 (496-547), désireux de juger de l'intelligence respective de ses fils (Gao Yang était le deuxième), leur donna à démêler une pelote de fil très embrouillée. Yang, tel Alexandre à Gordes, la trancha de son épée. Ayant succédé à son aîné Gao Cheng 高澄 comme chef de la famille, et ayant fondé la dynastie de Qi en forçant le dernier empereur des Wei orientaux à abdiquer en sa faveur, il se montra d'abord bon souverain et, comme son père, chef de guerre de première valeur.

C'est sous l'année 554 que l'historien relate un fait qu'il signale comme ayant marqué le début de sa folie. Lors d'une campagne au Shanxi, il fit mettre à mort un officier qui avait failli et donna à ses hommes l'ordre de le manger. L'anthropophagie n'étais pas très rare en soi dans la Chine ancienne, mais dans ce cas particulier, Gao Yang alla bien au-delà des " usages » en ordonnant aux soldats de faire disparaître jusqu'aux os et excréments.

L'anecdote la plus « pittoresque » est celle où on le voit, en 555, décapiter sa favorite sur un coup de colère, puis, ayant convié ses ministres à un banquet, sortir de sa manche sa tête, sous le regard horrifié de ses convives, enfin faire dépecer son corps, se saisir d'une cuisse et faire mine de jouer de la mandoline (pipa) avec, et s'écrier pour finir, en pleurant : «Jamais je ne retrouverai une beauté pareille. »

Une anecdote d'un intérêt particulier, car elle illustre le bouddhisme assez curieux de Gao Yang, nous le montre pratiquant la cérémonie de la " libération des êtres vivants » (fangsheng 放生), consistant à libérer oiseaux ou poissons et tenue normalement lors d'un des six jours de retraite mensuels (zhai 齋). Cette fois-là, Gao Yang monta sur une haute terrasse, d'où il fit précipiter dans le vide des prisonniers « libérés » qu'il avait fait affubler d'ailes en bois, éclatant de rire chaque fois que l'un d'eux s'écrasait au sol. Il avait par ailleurs fait dresser dans la cour du palais un enclos dans lequel étaient enfermés des condamnés à mort, qu'il massacrait de ses propres mains avec un luxe de cruauté quand l'envie lui en prenait, presque quotidiennement à ce qu'il paraît. On n'en finirait pas d'énumérer les autres preuves de sa cruauté. Une dernière, cependant : étant un jour sorti incognito pour prendre l'humeur du peuple (Haroun al Rachid n'était pas le seul à procéder ainsi : on rapporte le fait à propos de plusieurs souverains chinois, qui cependant en général chargeaient d'autres de ce genre d'enquête), il demanda à une vieille femme rencontrée dans la rue ce qu'elle pensait de l'empereur. Elle lui répondit : «C'est un fou » et il la tua sur le champ.

Un trait remarquable de son caractère est l'exhibitionnisme. Celui-ci était apparu assez tôt, quand, encore jeune marié et à la grande surprise de sa femme, il se mettait torse nu pour bondir et courir interminablement. Il se confirma en 553, quand, menant ses troupes contre les Khitan, en une chevauchée de près de cinq cents kilomètres, torse nu et cheveux au vent. Par la suite, on le voit parcourir la capitale sur les animaux les plus divers, bœufs, ânes, chameaux et éléphants, qu'il monte à cru. Il avait fait dresser dans l'un de ses palais deux échafaudages sur lesquels reposait un tronc d'arbre, sur lequel il s'amusait à courir, danser et virevolter, tant et si bien que « les spectateurs en avaient le cœur glacé ».

Selon le Bei-Qi shu, c'est en grande partie l'alcool (de l'abus duquel il devait d'ailleurs mourir) qui était le principal responsable de ses débordements. Une anecdote saisissante nous le montre se glissant sous le lit de sa mère et le soulevant de ses épaules pour la dérider, car elle était en colère contre lui. Sa mère étant tombée et 
s'étant blessée, il fait dresser un bûcher et veut se jeter dans le feu. Sa mère ayant eu le plus grand mal à l'en empêcher, il exige de recevoir le fouet, puis en pleurant jure qu'il ne boira plus, ce qui est bien sûr un serment d'ivrogne. Nous avons soumis ce texte à un médecin alcoologue qui l'a jugé par son luxe de détail presque conforme aux exigences modernes d'un rapport clinique.

Pour conclure, Li Baiyao nous dresse de Gao Yang et de ses troubles mentaux une image cohérente jusque dans les détails, et qui plaide en faveur de la fiabilité du Bei-Qi $s h u$, et plus généralement des historiens chinois médiévaux, au contraire de l'idée trop répandue que leurs ouvrages ne sont que des tissus de lieux communs, en particulier justement en ce qui concerne les empereurs fous. Dans le cas de Gao Yang, ses actes sont décrits avec une trop grande précision ${ }^{1}$ et une trop grande cohérence entre eux pour que l'on puisse y soupçonner des inventions, ou même de simples exagérations.

\section{Une capitale de la naissance à la mort : Nankin sous les Six Dynasties}

Poursuivant notre étude de la Nankin médiévale, on s'est consacré cette année à la rivière Huai, qui la traversait, et en particulier à un phénomène caractéristique qui l'affectait fréquemment. C'était une sorte de mascaret, ou plutôt de grande vague causée par une montée soudaine du fleuve et aggravée par son rétrécissement brusque en amont. En heurtant le promontoire de Shitou 石頭, le flot rejeté remontait la Huai et causait de graves dégâts, détruisant un grand nombre de bateaux, emportant souvent le fameux pont de bateaux (voir sur ce pont notre rapport de 2007-2008) et inondant les quartiers bas de la capitale, en faisant de nombreuses victimes. On a relevé dans les histoires dynastiques des Jin, des Song, des Qi et des Liang, ainsi que dans l'Histoire $d u$ Sud (Nanshi 南史) tous les passages relatifs à ce phénomène, aux destructions qu'il occasionnait, aux secours organisés par les autorités, etc. On a constaté que les annales et les mémoires sur les cinq éléments étaient loin de mentionner toutes les occurrences de ce phénomène, parfois annuel, parfois ne se reproduisant qu'après de longues années et on a pu en déduire que n'étaient rapportés dans les textes officiels que les évènements interprétés par les historiens comme les présages d'un grave évènement (défaite, mort d'un empereur, etc.) ou sanctionnant après coup une déficience politique de la part des empereurs et de leurs gouvernements. Les périodes d'occurrence fréquente correspondant à des périodes de trouble, et celles où il n'est pas signalé, à des périodes de calme, ou à des périodes desquels, pour des raisons diverses, les ministres n'osaient pas faire au souverain les remontrances suscitées par les phénomènes naturels, et, par voie de conséquence, leur notation par les historiens.

Cette étude a donné lieu à une intervention dans le colloque EPHE-Collège de France de juin 2009 ainsi qu'à une publication.

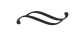

1. On peut encore évoquer à cet égard le récit, hallucinant de réalisme, de la manière infiniment cruelle dont, en 558, il fit tuer deux princes, ses frères (Zizhi tongjian, 558-40). Là encore, on assiste à des revirements d'humeur qui semblent lui avoir être propres, des désirs de pardon et des mouvements de pitié qui se retournent d'un seul coup en pulsions meurtrières : un autre trait de caractère bien connu des psychiatres et des alcoologues. 
Programme de l'année 2009-2010 : I. De la difficulté du portrait : Liang Yuandi (Xiao Yi, 508555). - II. Une capitale de la naissance à la mort : Nankin sous les Six Dynasties (suite).

\section{De la difficulté du portrait : Liang Yuandi (Xiao Yi, 508-555)}

Après une année consacrée à ce cas pathologique - c'est même le mot de monstre qui vient à l'esprit - que fut Gao Yang 高洋 (529, regnavit 550-559), premier empereur des Qi du Nord 北齊, nous avons consacré cette année à son homologue méridional et quasi-contemporain, Xiao Yi 蕭繹 $(508, r$. 552-555), septième fils de l'empereur Wu de Liang, qui ne lui est guère inférieur en perversion et cruauté.

Cette façon de le présenter pourra surprendre, voire choquer, ceux, somme toute assez nombreux, à qui le nom est familier. Mais si nous avons donné comme soustitre à cette conférence « De la difficulté du portrait », c'est bien parce que Xiao Yi, connu des historiens de la littérature comme un poète de valeur et le destinataire de la fameuse 《Lettre au prince de Xiangdong » 與湘東王書 dans laquelle son demifrère Xiao Gang 蕭綱 (empereur Jianwen, 503, r. 549-551) a développé ses idées sur la poésie, et aussi comme l'auteur du « Maître de la tour d'or» (Jinlou zi 金樓子), un ouvrage d'une grande originalité, bien connu aussi des historiens de la peinture comme l'auteur de la magnifique peinture connue sous le nom de « Rouleau des ambassadeurs » (ou, plus littéralement, des porteurs de tribut, Zhigong tu 職貢圖), bien connu enfin des spécialistes de l'histoire du livre comme le bibliophile et le collectionneur le plus acharné de son temps. Cependant, il n'est, ou ne semble, connu de chacun qu'à la lumière de son domaine propre, tous ayant en commun de tout ignorer, ou en tous cas de dire rien ou très peu, de ce qui est du ressort des autres spécialités, ainsi que du personnage historique, sur lequel pourtant les documents ne manquent pas. On a essayé cette année de combler cette lacune, en tentant une approche synthétique d'un personnage assez peu sympathique, mais certainement remarquablement doué, qui mérite sans conteste (non point moralement, mais en tant qu'objet historique) d'être mieux connu qu'il ne l'est. Nous présenterons ici les résultats de notre étude dans un ordre qui ne fut pas forcément celui de la conférence.

On a commencé notre étude par un relevé complet des ouvrages qui lui sont attribués dans les différentes sources, essentiellement les chapitres bibliographiques du Livre des Sui (Suishu 隋書). On trouve des ouvrages historiques, taoïsants, un traité d'art militaire, etc. À l'exception du Jinlou zi, seuls en ont parfois survécu les préfaces ou des fragments épars. On a examiné ensuite la liste de ses œuvres courtes en prose et de ses poèmes dans les collections de Yan Kejun et Lu Qinli. Ce fut en particulier l'occasion de présenter aux étudiants les différents genres en prose.

On a consacré la meilleure part de nos efforts à son Jinlouzi qui, malgré des traits bien remarquables, n'a jamais été étudié systématiquement ${ }^{1}$. Ce livre comporte

1. Il faut signaler cependant un article de notre éminent collègue japonais Kôzen Hiroshi (dont on peut rappeler ici qu'il enseigna jadis une année entière chez nous, en 1985-1986, sur l'invitation de notre collègue Jean-Pierre Diény), «La vie de Xiao Yi et le Jinlouzi 蕭繹的生活與金樓子》, dans Yiyu zhi yan 異域之眼, 2001, p. 154-168. Il s’y penche surtout sur la septième section du livre, « S'établir par la parole (liyan 立言) », mais nous venons d'apprendre par lui-même qu'il a terminé une traduction complète de l'ouvrage, qui sera publiée prochainement. Nous avons utilisé la compilation de fragments du Xuebuzu zhai congshu 學不足齋叢書 de Bao Tingpo 鮑廷博, dans l'édition reproduite par la maison Chûbun de Kyôto. 
quatorze sections, artificiellement arrangées en six chapitres, dont seules deux ou trois ont quelque chance d'être complètes. Nous avons lu en tout ou partie les plus dignes d'intérêt.

On a commencé par la quatorzième et dernière section, « Sur moi-même » (zixu 自序), dans lequel, comme son titre l'indique, le prince nous parle de lui. Les souvenirs intimes, parfois fort touchants, en font un document particulièrement intéressant, car d'une nature rarissime dans les sources médiévales. Il s'ouvre sur cette petite introduction :

Combien éphémère est la vie humaine! Elle est comme la flamme qu'on entrevoit par un trou foré dans la pierre, comme l'éclair aperçu à travers une fissure. Elle est semblable à la luciole qui meurt en voyant l'aube, à la rosée qui disparaît quand se montre le soleil. Comment pourrais-je ne pas parler de moi?

Ceci est déjà révélateur d'un trait de caractère de Xiao Yi. Alors que les images alignées ici font parti d'un stock de métaphores classiques issus d'un fonds ancien et ravivées par la littérature bouddhique, et proposent de ne voir dans la vie humaine qu'une illusion fugace, ce qui revient à inviter à ne pas parler de soi, Xiao Yi retourne le propos : c'est justement la réalisation de la brièveté de la vie qui justifiera ses épanchements personnels. Xiao Yi, on a mainte occasion de le vérifier, est un égocentriste.

On ne s'étonnera donc pas qu'il se montre souvent très satisfait de lui-même, comme quand il raconte son souvenir littéraire le plus ancien :

À cinq ans je pouvais composer un poème. Ayant reçu l'ordre ${ }^{1} \mathrm{~d}^{\text {'en }}$ composer un, je fis celui- ci : «Les lentilles sur l'étang à peine nées se joignent; les fleurs dans la forêt à peine ouvertes sont déjà denses. Quand la brise arrive les fleurs et les branches bougent. Quand le soleil brille, sa lueur flotte sur l'eau. » À partir de là, j'ai commencé à étudier l'art de composer un texte.

Ce poème n'a évidemment rien d'extraordinaire : c'est un tissu d'images toutes faites, telles que les jeunes aristocrates les apprenaient dans les thesauri poétiques qui connaissaient justement à cette époque un développement rapide. L'intérêt de l'anecdote est de jeter indirectement une petite lueur sur l'éducation des princes. On notera à cet égard que le schéma tonal (PPPZZ/PPZPP//PZPPZ/ZZZPP) est presque parfait : seul le deuxième caractère du deuxième vers l'empêche d'être conforme à ce qui sera le schéma classique des Tang. Ce document montre donc avec éloquence le degré de technique poétique que pouvait acquérir à l'époque un tout jeune enfant. L'étude que nous avions faite il y a quelques années de poèmes échangés entre le grand poète Shen Yue 沈約, initiateur de la poésie tonale, et le prince héritier Xiao Tong 蕭統 (Zhao-

1. Cet ordre venait probablement de son père, qui était lui-même un bon poète. On sait que les empereurs avaient peu de contacts intimes avec leurs enfants et que souvent, dans leur jeune âge, ils ne les voyaient guère qu'à l'occasion d'audiences publiques, au cours desquels ils leur faisaient subir de petits examens pour constater le développement de leurs connaissances. Sans doute est-ce le cas ici. On note que la scène, si les souvenirs de Xiao Yi sont exacts, se situe en 513, à une époque où l'empereur n'est pas encore entrée dans la période « bouddhiste » de sa vie, à une date où la poésie ne lui apparaît pas encore comme un passe-temps douteux (on pense à la réaction très négative qui est la sienne quand il apprend en 532 ou 533 que le prince héritier s'adonne à la poésie légère avec ses officiers.) 
ming Taizi 昭明太子), dont il était le précepteur, et plusieurs jeunes amis du prince, nous l'avait appris. Nous en avons ici une nouvelle confirmation. Xiao Yi composa le poème en question « à l'âge de cinq ans », donc en 513, l'année même de la mort de Shen Yue. Peut-être bénéficia-t-il, en tant que prince, de quelques leçons de la part du grand poète.

Un autre souvenir d'enfance, assez attendrissant, témoigne de sa passion précoce pour la lecture :

Quand j'étais petit, les soirs d'été, une fois abaissée la gaze pourpre de ma moustiquaire, avec auprès de moi un gobelet d'argent empli de vin doux de Shanyin 山陰, je lisais couché, le plus souvent jusqu'à l'aube.

D'autres souvenirs intéressants sont liés à ses rapports avec ses lecteurs, dont le mauvais état de ses yeux (il en perdit un assez tôt) nécessitait l'aide.

On trouve dans la même section plusieurs autres précieux aperçus de la vie des aristocrates médiévaux par exemple, une description assez concrète (et tout à fait unique), du jeu connu sous le nom de shefu et qui consistait à deviner, en s'aidant des techniques de la divination, des objets cachés sous un plat renversé, jeu où, prétend-il, il réussissait huit à neuf fois sur dix. Il en donne comme exemple un souvenir de jeunesse. Un de ses cousins avait ainsi caché un anneau d'or, un anneau d'argent et un anneau d'ambre. Xiao Yi « mania les brins d'achillée » et obtint l'hexagramme gou 循 (44), dont le premier trait, continu, représente le ciel. Il en déduisit la forme des objets à deviner et leur nature. Quant à leur matière, il les devina à partir d'éléments du commentaire traditionnel de cet hexagramme.

Un autre jeu consistait, également en partant d'un hexagramme, à deviner un caractère qui avait été écrit sur un papier ensuite replié. Un jour qu'il jouait avec quelques uns de ses officiers, l'hexagramme obtenu étant ding 鼎 (50), composé du trigramme li 離, qui correspond au soleil, en haut, et du trigramme xun 巽, qui correspond au bois, en bas, il en déduisit que le caractère caché était composé du caractère soleil 日 superposé au caractère bois 木, soit le caractère gao 杲, ce que lui confirma le fait que Gao était le nom personnel de d'un de ses compagnons. « Mes autres expériences, conclut-il, sont du même ordre. »

On peut rester sceptique quant au succès de ses déductions, mais la description qu'il nous donne de ces deux jeux n'en constitue pas moins un document unique.

Xiao Yi fut, on l'a dit, un grand bibliophile et les rouleaux en sa possession atteignirent le chiffre considérable de cent quarante mille (ce qui correspondait, nous dit-il dans ses souvenirs personnels, à environ dix mille titres). La sixième section du Jinlouzi, « Collectionner les livres » (jushu 聚書), se présente comme le carnet d'un collectionneur et constitue, là encore, un document absolument unique. Xiao Yi procède par ordre chronologique. Ses premiers livres lui furent offerts par l'empereur son père. Par la suite, dans tous ses postes, il fit acheter sur les marchés ou copier chez leurs propriétaires (parfois des supérieurs de monastères), d'innombrables textes de toute nature. Il reste fort discret sur la manière dont il entra en possession de certains fonds, dont la collection du grand érudit et amateur Liu Zhilin 劉之遴, connu pour sa collection de bronzes antiques, manière à propos de laquelle il dit simplement : « J'eus également des livres de Yue Yanchun 樂延春 et de Liu Zhilin, en tout près de cinq mille 
rouleaux. » Or, nous apprend la biographie de ce dernier dans le Livre des Liang, il le fit empoisonner alors qu'il remontait le fleuve - avec sa collection - pour se réfugier auprès de lui. Aussi, quand il déclare sans plus de précisions : «Yu Jue 虞爵 possédait environ cinq cents rouleaux anciens. Je les acquis tous », ou : " J'obtins aussi d'un habitant de la capitale provinciale, Zhu Danyuan 朱澹遠, qu'il me fît don de livres rares en sa possession », on peut s'interroger sur la manière dont il fit ces acquisitions. Hélas, tous ces trésors devaient partir en fumée, littéralement, quand par pur dépit (une preuve de plus de son égotisme) il mit le feu de sa propre main à cette bibliothèque unique, qui comprenait même des manuscrits des illustres calligraphes Wang Xizhi 王羲之 et Wang Xianzhi 王獻之, lors de la prise de Jiangling en 555 .

Pour en finir avec le Jinlouzi, il est à noter que les deux chapitres intitulés respectivement «Instructions à mes fils » 教子篇 et 《Instructions pour mes funérailles » 終制篇 ne présentent pas seulement une communauté de titres, mais aussi une proximité de contenu avec les très célèbres « Enseignements familiaux de maître Yan » 嚴子家訓 du grand lettré Yan Zhitui 嚴之推 (531-591). Ce n'est pas un hasard : Yan fut dans sa jeunesse un officier de Xiao Yi et la lecture de leurs ouvrages, ainsi que le relève Kôzen Hiroshi, révèle plus d'un point commun. Yan Zhitui, grand ami de l'étude, avait pour l'érudition de Xiao Yi une grande admiration et fait dans son ouvrage référence à lui à plusieurs reprises ${ }^{1}$.

Il fallait bien sûr se pencher aussi sur l'aspect le plus connu de Xiao Yi, sa production poétique. On a lu plusieurs de ses meilleurs poèmes, ceux qui figurent dans les Nouveaux chants des terrasses de jade (Yutai xinyong 玉臺新詠) de Xu Ling 徐陵. Ce fut aussi l'occasion, en lisant des poèmes composés en compagnie de son frère aîné Xiao Gang en réponse à leur lointain parent Xiao Zixian 蕭子顯, d'étudier le fonctionnement d'une joute poétique. On a exploré également des pans moins connus de sa production littéraire, en particulier le texte de la stèle commémorant la construction du Kiosque de la libération des êtres vivants 放生亭, à Jiangling (sur cette pratique, v. supra rapport 2008-2009). C'est une pièce où le sentiment bouddhique est pour ainsi dire anéanti par l'accumulation d'allusions historiques et littéraires que nous n'avons pu toutes identifier. C'est l'aspect le moins séduisant de son œuvre, mais il est bien représentatif de sa personnalité : une grande intelligence et une immense érudition, mais un esprit alambiqué et même retors, comme on le verra ${ }^{2}$.

L'étude menée sur Xiao Yi jusque là nous inviterait quand même plutôt à voir en lui un personnage assez attachant, quoique probablement tourmenté, si la lecture indispensable des textes le concernant dans le Livre des Liang et l'Histoire du sud, ne nous montrait un homme cruel jusqu'à la perversité, retors, d'une jalousie et d'une mesquinerie maladives.

Qu'il ait ordonné à son général Zhu Maichen 朱買臣, une fois la capitale reprise, de tuer l'ex-empereur Xiao Dong 蕭棟, un enfant qui n'avait été que l'homme de paille de Hou Jing 侯景, pourrait encore passer pour de la realpolitik, bien que l'exécu-

1. Op. cit. supra, n. 1, p. 259

2. Dans le même ordre d'idées, on trouve dans son recueil une douzaine de poèmes sur les noms d'oiseaux, de bâtiments, de véhicules, de districts, les titres de chanson, etc., qui ne consistent qu'en de savantes accumulations d'allusions et de jeux de mots. Ces pièces dont le caractère ludique est évident, ont sans doute été composé à l'occasion de joutes poétiques. 
tion de l'ordre se soit effectuée comme un vulgaire assassinat (Zhu Maichen précipita Xiao Dong, ainsi que son frère, dans le fleuve, après les avoir invités à venir boire une coupe de vin sur son vaisseau-amiral). Impardonnable en revanche est d'avoir laissé les fils de son frère Xiao Ji 蕭紀 mourir de faim dans leur prison.

Mais ce sont peut-être des actes moins graves qui sont le plus révélateurs de sa bassesse d'esprit. Ainsi, quand il apprit la mort de son frère Xiao Lun 蕭綸, qui était un obstacle sur la route du pouvoir, il sauta de joie sur place au point de fendre ses sandales de bois. Plus tard, quand il apprit que son autre frère, le prince de Wuling Xiao Ji 蕭紀, qui s'était rendu indépendant à Chengdu, descendait dans l'Est, dans l'intention évidente de s'emparer de l'empire, s'il n'était pas illégitime qu'il s'opposât à lui, mais il se livra à cette occasion à un acte que l'historien, qui par nature ne sympathise guère avec la superstition, réprouve (ce que montre le seul fait qu'il le mentionne) : il fit exécuter par un magicien un portrait de Ji, sur lequel il lança des fléchettes afin de lui jeter un sort mortel (il était lui-même assez bon peintre pour faire de son demifrère un portrait ressemblant, mais sans doute en cette occurrence la ressemblance du portrait importait moins que la charge néfaste que seule un magicien pouvait y introduire $^{1}$.) Plus tard, après la mort de $\mathrm{Ji}$, tué par un de ses généraux, il lui fit donner un nom posthume pour le moins étrange : Taotie 饕䬸. Taotie est le nom du glouton des antiques légendes, animal insatiable. Est-ce pour dire que son frère, en désirant l'empire, avait fait preuve d'un appétit excessif? Toujours est-il qu'en lui donnant ce nom, il fit preuve, en même temps que de son goût pour l'érudition et les choses antiques, d'un esprit pour le moins mesquin, mais inventif certes, puisque le nom de Taotie ne figure pas sur la liste des noms posthumes, laquelle est une catégorie fermée.

Xiao Yi était d'une jalousie maladive. L'Histoire $d u$ Sud dit que «si quelqu'un l'emportait sur lui en quelque domaine, il le faisait tuer ». Et si le Livre des Liang nous dit qu'il fit empoisonner Liu Zhilin pour s'emparer de ses livres, l'Histoire du Sud nous dit qu'il le fit parce qu'il était envieux de son talent. Les deux explications, d'ailleurs, ne s'excluent nullement. La mesquinerie de Xiao Yi atteint parfois des proportions stupéfiantes. Une princesse de ses tantes avait épousé Wang Lin 王琳, qui devait s'illustrer comme général. Ils avaient eu ensemble huit enfants, tous superbement doués. Xiao Yi, qui n'avait pas eu beaucoup de succès avec ses propres enfants, fut si jaloux de lui qu'il obligea Wang Heng 㹸, le frère aîné de sa favorite du moment, à changer son nom en Lin, de manière à introduire un doute dans les esprits des gens quant à l'identité du père des petits génies !

Pour couronner le tout, on peut évoquer la mort atroce de Wang Wei, un acolyte du rebelle Hou Jing capturé lors de la débâcle de ce dernier, à qui il avait cependant envisagé de faire grâce, jusqu'à ce qu'on lui signalât un texte dans lequel Wang Wei s'était moqué de son œil borgne. Alors il le fit clouer par la langue à un pilier de la salle du trône, lui fit ouvrir le ventre et ordonna aux parents de ses victimes de le dépecer, encore vivant.

1. Un autre trait caractéristique de Xiao Yi est qu'il semble avoir été fort superstitieux. Par exemple, après la mort de son père, il fit sculpter une statue à sa rassemblance et la fit placer dans ses appartements afin de pouvoir prendre conseil d'elle en toutes circonstances. 
En conclusion, on ne peut que souscrire au jugement de l'Histoire du Sud (Beishi 北史, biographie de son fils Xiao Dahuan 蕭大歡), qui le dit cruel et méfiant, et de celui que porte indirectement sur lui le Livre des Liang, disant, à propos d'un autre de ses fils, Xiao Fangju 蕭方矩, qu'il était encore plus cruel et violent que son père. On est bien loin de l'aimable l'enfant amoureux des livres.

On peut s'étonner à propos du jugement porté par l'Histoire du Sud que le Livre des Liang (hormis le jugement indirect mentionné ci-dessus), soit dans l'ensemble beaucoup plus indulgent à l'égard de Xiao Yi. Il y est, par exemple, dit fort peu de choses de sa mère, alors que l'Histoire du Nord rapporte qu'elle n'était à l'origine qu'une femme du palais de rang très bas et que, un jour qu'elle apportait la literie dans la chambre de l'empereur, un courant d'air soudain souleva sa jupe, Xiao Yan s'était jeté sur elle pour assouvir un désir tout aussi soudain. L'anecdote a, il est vrai, un parfum de médisance. La différence de traitement entre ces deux sources s'explique sans doute, ainsi que le propose Kôzen Hiroshi ${ }^{1}$, par le fait que le Livre des Liang fut écrite par Yao Silian 姚思廉, dont le père avait servi les Liang : il eût de ce fait été, bien qu'indirectement, impie de trop dénigrer un des leurs. En revanche, les auteurs de l'Histoire du Nord n'avaient pas à prendre de telles précautions.

Bien des points dignes d'intérêt restaient à étudier dans la vie et dans l'œuvre de Xiao Yi, mais il est apparu que l'ensemble de l'auditoire était hostile à l'idée de poursuivre l'étude du personnage. On a donc remis la poursuite de ce travail à plus tard. Une publication est en cours de préparation.

Au cours de notre étude sur Xiao Yi, nous avons été amenés à lire quelques poèmes palindromes, ce qui nous a amené à traiter un peu de ce genre ludique et nous a permis un bien agréable détour par le "Poème du diagramme cosmique » (Xuanji tu shi 璇璣圖詩) de Su Ruolan 蘇若蘭, une femme du IV ${ }^{\mathrm{e}}$ siècle, sans doute le plus étonnant palindrome de l'histoire littéraire mondiale. Ce poème, envoyé, sous forme d'une broderie, à un époux qui la négligeait, se présente comme un carré de vingt neuf caractères de côté, soit 841 caractères en tout, susceptibles d'un nombre incalculable (certains l'ont estimé à plusieurs milliers) de lectures horizontales, verticales, diagonales, en carré, etc., toutes rimées, le tout étant compliqué par le fait que la broderie originale, évidemment perdue depuis longtemps, comportait des circuits colorés sur lesquels on a malgré tout gardé quelques indications. Pour donner une idée de son raffinement, autour du caractère central « cœur » (xin 心), c'est-à-dire à la fois « centre du poème » et « amour », un premier carré de neuf caractères se lit : « Le poème du diagramme cosmique a enfin apaisé, de la dame Su le cœur » (en terminant sur le caractère central). Le point le moins intéressant à propos de ce poème n'est pas qu'il ait attiré l'attention de la fameuse impératrice Wu Zetian 武則天 des Tang. Une publication est prévue.

\section{Une capitale de la naissance à la mort : Nankin sous les Six Dynasties (suite)}

On a passé beaucoup moins longtemps sur ce second sujet, en mettant un point final à une étude commencée l'année précédente sur le temple des ancêtres de Jian-

1. Op. cit. supra, n. 1, p. 259. 
kang, la Nankin des Six Dynasties. On a en particulier étudié les dégâts que lui occasionnait parfois le «mascaret» de la Huai (cf. sup. rapport 2008-2009) : une malencontreuse divination du pourtant illustre Guo $\mathrm{Pu}$ 郭璞, $\mathrm{au} \mathrm{IV}^{\mathrm{e}} \mathrm{s}$., l'avait fait construire précisément dans la zone inondable (les bateaux étaient parfois amarrés à ses piliers!). Il dut être l'objet de fréquentes réparations, jusqu'à ce que l'empereur Wu des Liang le fît reconstruire à très grands frais, en 535, sur une terrasse surélevée. On apprend, au détour des textes, que, quand les inondations le menaçaient, les ministres devaient se rendre sur les lieux revêtus de leur armure, sans doute parce que le temple, représentait, en tant que lieu des sacrifices faits aux ancêtres des souverains régnants, un symbole de l'État, l'irruption des eaux étant alors assimilée à l'attaque d'une armée ennemie. 